LA WAENCE LIWEAMOAE NATIONAL LABDAATORY

\title{
Searching for Auxetics with DYNA3D and ParaDyn
}

W. G. Hoover and C. G. Hoover

September 2004

Workshop on "Auxetics and Related Systems" June 27-30, 2004 Poznan, Poland 
This document was prepared as an account of work sponsored by an agency of the United States Government. Neither the United States Government nor the University of California nor any of their employees, makes any warranty, express or implied, or assumes any legal liability or responsibility for the accuracy, completeness, or usefulness of any information, apparatus, product, or process disclosed, or represents that its use would not infringe privately owned rights. Reference herein to any specific commercial product, process, or service by trade name, trademark, manufacturer, or otherwise, does not necessarily constitute or imply its endorsement, recommendation, or favoring by the United States Government or the University of California. The views and opinions of authors expressed herein do not necessarily state or reflect those of the United States Government or the University of California, and shall not be used for advertising or product endorsement purposes.

This work was performed under the auspices of the U.S. Department of Energy by University of California, Lawrence Livermore National Laboratory under Contract W-7405-Eng-48. 


\title{
Searching for Auxetics with DYNA3D and ParaDyn
}

\author{
Wm. G. Hoover \\ Department of Applied Science \\ University of California at Davis/Livermore \\ and Methods Development Group \\ Lawrence Livermore National Laboratory \\ Livermore, California 94551-7808 \\ C. G. Hoover \\ Methods Development Group \\ Lawrence Livermore National Laboratory \\ Livermore, California 94551-7808
}

(July 26, 2004)

We sought to simulate auxetic behavior by carrying out dynamic analyses of mesoscopic model structures. We began by generating nearly periodic cellular structures. Fournode "Shell" elements and eight-node "Brick" elements are the basic building blocks for each cell. The shells and bricks obey standard elastic-plastic continuum mechanics. The dynamical response of the structures was next determined for a three-stage loading process: (1) homogeneous compression; (2) viscous relaxation; (3) uniaxial compression. The simulations were carried out with both serial and parallel computer codes-DYNA3D and ParaDyn - which describe the deformation of the shells and bricks with a robust contact algorithm. We summarize the results found here.

\section{INTRODUCTION}

Auxetic behavior-negative Poisson's ratio-is unusual but real. Relatively porous solids, such as the copper foam shown in Fig. 1 are auxetic [1]. Mesoscopic models for such microstructures, based on regular arrays of elements, can be auxetic too $[2,3]$. The models considered so far have generally been relatively simple, so as to facilitate theoretical analyses. Here we consider a more complex class of models, well-suited to numerical simulation and exploration. The underlying fulldensity "matrix material" is simple, obeying Hookean linear elasticity (Young's modulus $E=100$ and Poisson's Ratio $\nu=0.25)$ up to a yield strength $(Y=0.1=E / 1000)$, beyond which the continuing response to deformation becomes "plastic" ( and "irreversible").

By building up nearly-regular cell structures using this idealized elastic-plastic material we can create highly irregular compressed structures. When these structures are relaxed, to a local energy minimum, further longitudinal deformation can be analyzed for auxetic behavior. That is, squeezing in the $x$ direction should result in proportional shrinkage in the $y$ and $z$ directions. Because a wide range of materials and cell structures give rise to auxetic behavior in the laboratory we expected that relatively simple models should do so too. This paper describes our search for auxetic behavior. In Sec. II we review the microscopic approach, which points out the need for a macroscopic continuum approach to auxetic materials.
Sec. III is a review of the underlying continuum mechanics. Sec. IV describes the details of the numerical solution of the continuum equations. Sec. V describes our choices of initial and boundary conditions. Sec. VI is devoted to results and Sec. VII to our conclusions. Sec. VIII is a well-deserved acknowledgment of some of the debts we have incurred to the workshop organizer, Krzysztof Wojciechowski. Kris facilitated and supervised both the social and the scientific aspects of the stimulating workshop at which our material was presented.

\section{MICROSCOPIC STATISTICAL MECHANICS AND MOLECULAR DYNAMICS}

No doubt the fundamental basis of macroscopic thermodynamic and hydrodynamic behavior is microscopic, as was detailed by Boltzmann and Gibbs. They developed "statistical mechanics". The statistical mechanics of atomistic mass$m$ particles, interacting with pairwise potential energies $\phi(r)$ which provide central forces $F(r)$ and a dynamics, $m \ddot{r}=F$, which can be simulated with standard integration methods, was worked out independently by these two men. Further elaborations of their ideas were made necessary when fast computers, able to simulate the many-body problem, became available around the time of the Second World War. Fermi, Alder, and Vineyard were responsible for pioneering simulations in the new field of "molecular dynamics". For a brief history see the review by Hoover [4].

By 1967 computers were able to solve deformation problems involving the correlated motions and interactions of a few thousand particles. Shockwaves converting a cold solid into a hot fluid were simulated [5]. Simpler problems were considered. In considering them, it was natural to choose to describe the existing macroscopic formalism for elasticity in a form suitable for evaluation using molecular dynamics. Because molecular dynamics describes temperature (in terms of the kinetic energy of the moving particles) a description of the temperature dependence of the elastic response to strain became possible. In 1969 Hoover, Holt, and Squire developed a thermal microscopic description of the elastic constants in terms of the classic static-lattice sums (which they called the "Born" terms in recognition of Max Born's contributions to 
elasticity) along with "fluctuation terms" taking thermal effects into account $[6,7]$.

By 1989 it was feasible to carry out simulations with as many as a million atomistic particles [8-10]. Elastic-plastic indentation problems, in two and three dimensions, with both pair and many-body potentials, were carried out then. By 1992 realistic simulations of the indentation of silicon appeared. Fig. 2 is taken from the cover of the March/April 1992 issue of Computers in Physics. In the figure the indentation pits obtained using atomistic and continuum versions of a tetrahedral indentor are compared. In both cases the solid indentor penetrates a fully-atomistic workpiece. Fig. 3 shows two-dimensional simulations of precision metal cutting, from this same era [10]. Several of these early simulations represented metals by using the many-body embedded-atom potential [11]. In all of this early work careful attention was paid to the development of useful boundary conditions and to the analysis of quantitative measures of the simulation results. Even in two dimensions (and certainly in three) it was readily apparent that problems involving the plastic deformation of macroscopic bodies were best treated by continuum mechanics. Molecular dynamics takes too long. Not only the spatial and temporal limitations of molecular dynamics, but also the lack of fundamental information on atomistic forces, suggest still today that the macroscopic approach will be with us for the forseeable future. We turn to the simplest form of the macroscopic description in the next Section.

\section{CONTINUUM DYNAMICS}

Continuum dynamics describes the time development of matter with continuously differentiable velocities $v$, stress tensors $\sigma$, and energy densities e. The fundamental (partial differential) equation describing the motion of a continuum comes from momentum balance [12]. It gives the acceleration field in terms of gradients of the local stress-tensor components:

$$
\rho \dot{v}=\nabla \cdot \sigma .
$$

The "comoving" time derivative $\dot{v}$ is the time rate of change of velocity $v$ for a particular volume element. In principle the volume element is infinitesimal. Such a derivative is also the natural choice for a numerical method which divides the continuum into small but finite "Lagrangian zones" (or "finite elements") so as to reduce the number of degrees of freedom to a manageable number.

A well-posed continuum problem requires a relation giving the stress tensor $\sigma$ for the material in terms of the other state variables (energy, density $\rho$, strain $\epsilon, \ldots$ ). For simplicity we begin by considering an elastic equation of state, with stress proportional to strain. It is usual to write this equation of state in terms of the Lamé constants $\lambda$ and $\eta$ or in terms of the Bulk and Shear Moduli $B$ and $G$,

$$
B=\lambda+\frac{2}{3} \eta ; G=\eta \text {. }
$$

The linear elastic equation of state relates stress to the strains, which are symmetrized deformation gradients:

$$
\epsilon_{x x}=d u_{x} / d x ; \epsilon_{y y}=d u_{y} / d y ; \epsilon_{z z}=d u_{z} / d z ;
$$

$$
\begin{aligned}
& \epsilon_{x y}=d u_{x} / d y+d u_{y} / d x ; \\
& \epsilon_{y z}=d u_{y} / d z+d u_{z} / d y ; \\
& \epsilon_{z x}=d u_{z} / d x+d u_{x} / d z .
\end{aligned}
$$

For large deformations the extent of deformation $u$ becomes ambiguous. For that reason, in practical calculations the time derivatives of these equations (expressing the strain rates in terms of velocity gradients) are integrated instead. The stresses (integrated stress rates) from these strain rates need to be defined so as to be symmetric also. A symmetric stress tensor is necessary for a mechanically stable treatment of rotation [13]:

$$
\begin{gathered}
\sigma_{x x}=2 \eta \epsilon_{x x}+\lambda\left(\epsilon_{x x}+\epsilon_{y y}+\epsilon_{z z}\right) \\
\sigma_{y y}=2 \eta \epsilon_{y y}+\lambda\left(\epsilon_{x x}+\epsilon_{y y}+\epsilon_{z z}\right) \\
\sigma_{z z}=2 \eta \epsilon_{z z}+\lambda\left(\epsilon_{x x}+\epsilon_{y y}+\epsilon_{z z}\right) \\
\sigma_{x y}=\sigma_{y x}=\eta \epsilon_{x y} \\
\sigma_{y z}=\sigma_{z y}=\eta \epsilon_{y z} \\
\sigma_{z x}=\sigma_{x z}=\eta \epsilon_{z x}
\end{gathered}
$$

Now consider an application of elastic theory to the characterization of auxetic materials. Compress an otherwise stressfree cylinder in the $x$ direction. By setting the transverse stresses $\sigma_{y y}$ equal to zero, and assuming cylindrical symmetry, $\left(\epsilon_{y y}=\epsilon_{z z}\right)$, we can relate the definition of Poisson's Ratio $\nu$ (minus the ratio of the transverse to the longitudinal strain) to the Lamé constants and the moduli:

$$
\begin{gathered}
\nu=-\epsilon_{y y} / \epsilon_{x x}=\lambda /(2 \eta+2 \lambda) ; \\
B=\lambda+(2 / 3) \eta ; \\
\nu=\left(B-\frac{2}{3} \eta\right) /\left(2 B+\frac{2}{3} \eta\right) .
\end{gathered}
$$

Poisson's ratio is restricted in range by the requirements that both the Bulk and Shear moduli be positive:

$$
0<B, G<\infty \longrightarrow-1<\nu<\frac{1}{2}
$$

Equivalently, the fraction $\lambda / \eta$ must exceed $\frac{-2}{3}$ :

$$
B>0 \longrightarrow \frac{\lambda}{\eta}>\frac{-2}{3} \text {. }
$$

Typical values of $\frac{B}{\eta}$ and $\nu$ are:

$$
\frac{B}{\eta}=\left\{\frac{1}{3}, \frac{2}{3}, \frac{3}{3}, \frac{4}{3}, \frac{5}{3}\right\} \longrightarrow \nu=\left\{\frac{-10}{40}, \frac{0}{40}, \frac{+5}{40}, \frac{+8}{40}, \frac{+10}{40}\right\} .
$$

We use the simplest model for plasticity, in which an effective shear stress $\sigma_{\text {eff }}$ cannot exceed a fixed (constant) yield strength $Y$ :

$$
\begin{gathered}
\sigma_{\text {eff }} \equiv\left[\frac{1}{2}\left(\sigma_{x x}-\sigma_{y y}\right)^{2}+\frac{1}{2}\left(\sigma_{y y}-\sigma_{z z}\right)^{2}+\frac{1}{2}\left(\sigma_{z z}-\sigma_{x x}\right)^{2}+\right. \\
\left.2 \sigma_{x y}^{2}+2 \sigma_{y z}^{2}+2 \sigma_{z x}^{2}\right]^{\frac{1}{2}}<Y .
\end{gathered}
$$

With the specification of Young's modulus, Poisson's ratio, and the von Mises' yield strength, the constitutive properties of a simple "elastic-perfectly plastic" model are complete. 


\section{DISCRETIZATION OF THE CONTINUUM IN DYNA3D AND PARADYN}

The serial and parallel computer codes, DYNA3D and ParaDyn, need a list of nodes and a list of elements, a connectivity (nearest-neighbor nodes) list, as well as initial and boundary conditions. Each of the nodes has its own coordinates, which can vary with time. Each element is made up of one or more nodes. We describe the continuum structure in terms of simple elastic "shell" or "brick" elements. Each shell element corresponds to four nodes. Bricks require eight nodes. We choose an initial brick size $1 \times 1 \times 1$. For convenience we choose the size of the individual shells to be $1 \times 1 \times 0.1$, where the shell thickness 0.1 is again arbitrary. The shells can be treated by a wide variety of models. We have used two of the most popular choices available in DYNA, Hughes-Liu and Belytschko-Tsay. The shells are arranged in "panels" of $2 \times 2,4 \times 4,8 \times 8, \ldots$ shells. Our models based on brick elements have cubic vacancies.

Both kinds of meshes can be generated easily. An $n \times n \times n$ array of nodes without any vacant portions corresponds to $3 n(n-1)^{2}$ shells or $(n-1)^{3}$ bricks. It is simplest to generate all of these elements first, and then to discard from the list those necessary to make a regular array of vacancies. It is quite practical to consider vacancies covering the same volume as do $2^{3}, 4^{3}, 8^{3}$, and $16^{3}$ elemental bricks. The elements are initially arranged in periodic arrays such as the two shown in Fig. 4. Prior to deformation small random displacements, of order one percent of the shell or brick size, are applied to all of the nodes.

"Contact algorithms" prevent or limit the interpenetration of elements. This can be accomplished by Lagrange Multipliers (which enforce the corresponding geometric constraints) or by using conditional Hooke's Law springs (which force any overlapping elements to separate). We have used both approaches in our simulations and found no significant differences. Likewise, for the two distinct shell-element models, we found no significant differences between them.

\section{DEFORMATION OF THE MODEL}

We deform the model by applying accelerations to all of the surface nodes in a "compression phase". The accelerations are chosen to be proportional to the initial separation of the node from the center of mass, and to a piecewise linear function of a reduced time variable, chosen here to vary from -2 to 2 :

$$
\begin{aligned}
& -2<t<-1 \longrightarrow \ddot{r} \propto r(0)[+t+2] ; \\
& -1<t<+1 \longrightarrow \ddot{r} \propto r(0)[-t+0] ; \\
& +1<t<+2 \longrightarrow \ddot{r} \propto r(0)[+t-2] .
\end{aligned}
$$

See Fig. 5 for a summary of the displacements and velocities which accompany these accelerations. In most cases we have arbitrarily chosen the time scaling and proportionality constant so that the side length at $t=+2$ is ten percent less than that at $t=-2$ for shells, and twenty percent less for bricks. The compression is carried out over a period of several sound traversal times.
Once this plastic deformation has been carried out a second "relaxation phase" is imposed on the structure. The relaxation is carried out by zeroing all the velocities at integral multiples of $100 d t$. This ad hoc procedure was chosen because DYNA3D does not have a dynamical damping which is useful for this class of problem. A third and final "deformation phase", in which the accelerations are applied only in the $x$ direction, with an amplitude 100 times smaller than those of the compression phase, is applied last of all. The response of the structure to this last deformation can then be analyzed to determine Poisson's ratio for the compressed and relaxed material.

Throughout, the motion is approximated by a second-order Runge-Kutta "midpoint" integration. For the two coupled ordinary differential equations $\dot{r}=v ; \dot{v}=a(r, v)$ this integration algorithm is as follows:

$$
\begin{aligned}
& r^{\prime}=r+v \frac{d t}{2} ; v^{\prime}=v+a(r, v) \frac{d t}{2} \\
& r=r+v^{\prime} d t ; v=v+a\left(r^{\prime}, v^{\prime}\right) d t .
\end{aligned}
$$

DYNA3D and ParaDyn simulations solve equations for the time development of the nodal coordinates and velocities, the stresses for each element (by integrating the corresponding strain-rate tensor in time and applying the von Mises plastic yield condition as needed), and the plastic strain for each element(proportional to the irreversible work done on that element). The detailed models for shell elements are relatively complicated [14]. Interpolations within brick elements are carried out with a simple isoparametric representation. Function values inside an analogous square, with $-1<x, y<+1$ would be computed as follows:

$$
\begin{gathered}
f_{x}^{y} \equiv f(x, y)= \\
\frac{1}{4}\left[f_{-}^{-}(1-x)(1-y)+f_{-}^{+}(1-x)(1+y)+\right. \\
\left.f_{+}^{-}(1+x)(1-y)+f_{+}^{+}(1+x)(1+y)\right] .
\end{gathered}
$$

It must be understood that the $x$ and $y$ coordinates in this interpolation are constants and do not vary with time.

The graphical data are plotted as the program progresses by a special program GRIZ. GRIZ reads the data files generated by DYNA3D or ParaDyn. Both DYNA3D and GRIZ are available from the United States Government's Energy Science and Technology Software Center in Oak Ridge, Tennessee. Typical results from the simulations appear in the next Section.

\section{RESULTS}

The most useful diagnostic tools for these problems were (1) animated views of the overall structure, (2) time histories of the coordinates for particular nodes, and (3) the time history of the kinetic energy. DYNA3D and ParaDyn allow for the use of several "materials" in a simulation, and the accompanying graphics package GRIZ allows the analyst to look at particular sets of materials. This makes it easy to see "cutaway" views of the structure, as well as cross sections. The simplest application of this feature is to term all those elements subject to external forces "Material 1" and the rest 
"Material 2". A graphic display, restricted to Material 2, then shows only the interior of the deforming material. See Figs. 6, 7, and 10 for examples.

Because the defining property of auxetic materials is their unusual response to longitudinal loading it is useful to compute the strains $\left\{\epsilon_{x x}, \epsilon_{y y}, \epsilon_{z z}\right\}$. Overall strains can be defined in terms of differences of boundary-particle coordinate sums (those on the positive $x$ face minus those on the negative $x$ face, divided by the original value of the difference of these sums, gives $\epsilon_{x x}+1$ ).

All of our simulations treated cubes of material subject to external loads on their faces. A better treatment would be to use periodic boundaries. That approach would provide smoother results, with reduced rate dependence. In a periodic deformation the central cube of material would be deformed by periodic images translated in space. These boundary conditions are the typical ones for molecular dynamics, but are not available options in most solid-mechanics computer codes.

We carried out quite a variety of simulations in searching for auxetic behavior. The first two weeks of our search were fruitless, but eventually the structures and procedures described here were successful. In this work we record only a few observations. It is quite practical to run simulations with some tens of thousands of elements on a work station. Typical runtimes on such a serial machine are of order several microseconds per element timestep. Thus a work-station run of 100,000 steps with $N$ elements would be executed in approximately $N$ seconds of computer time.

At Livermore it is quite feasible, though a bit tedious, to reduce these times by a factor of order 100, by assigning a simulation to several processors. Fig. 8 illustrates the assignment of a $65 \times 65 \times 65$-node problem to 32 processors. To check the sensitivity of the results we found we varied the Poisson's ratio of the matrix material, the rate of compression, the shear modulus, and the amplitude of the initial perturbations. Of all of these, the results are most sensitive to the perturbations. Fig. 9 shows the displacements of a typical node during the third-stage small deformation used to determine Poisson's Ratio for the deformed cellular material. The structure is shown in Fig. 10. The effective Poisson's ratio for this multishell structure is changed, from -0.1 to -0.4 , by a decrease in the random displacements from the range $\pm 2.5 \%$ to the range $\pm 0.5 \%$.

We carried out several simulations involving brick elements. See Figs. 7 and 11 for examples. Despite varying the thickness of the partitions between empty cells (one, two, or three bricks thick) and the size of the cells $(1 \times 1 \times 1$ to $40 \times 40 \times 40)$ we have not yet (midJuly, 2004) found auxetic behavior for the brick structures. In the structure shown in Fig. 11 the loads were applied directly to the outer nodes shown in the figure. In our earlier work the loads were applied to an outer complete skin of brick elements, but that boundary condition was more sensitive to hourglass instability, and we abandoned it. Because a large number of bricks can serve as a surrogate shell there is no doubt that we will eventually find auxetic behavior for the brick structures too.

\section{CONCLUSIONS}

Our simulations are insensitive to the numerical algorithms used to represent the underlying brick and shell elements, so that one can use the continuum codes with confidence. Individual cells made up of shell elements, when severely buckled, exhibit auxetic behavior. The details of this behavior, for the loading rates we used, are sensitive to the small perturbations from ideal symmetry used in the initial conditions. The most negative Poisson's Ratio found in our exploratory calculations (most of which used a matrix Poisson's Ratio of $\frac{1}{4}$ ) was -0.4 . See again the right side of Fig. 9.

We conclude that auxetic materials are an ideal problem for parallel computation. The problems are nonlinear and chaotic, with the results mostly not known in advance, and the simulations are relatively quick and inexpensive. We expect that the future will lead to a systematic understanding of auxetic behavior supported by computer simulations.

Overall, we conclude that DYNA3D and ParaDYN are useful research tools for predicting auxetic behavior. A number of improvements could be made to DYNA3D and to ParaDYN to facilitate further work. Periodic boundary conditions would be very useful for exploring the response of anisotropic complex structures. Isotropic cell structures (as opposed to those with cubic symmetry) could be developed. One way to do this would be to base a continuum mesh on the structure of a typical atomistic fluid, taken from a molecular dynamics simulation. Beam elements which prevent contact would be particularly welcome in modeling the auxetic foams remaining after the evaporation of nominally-spherical bubbles.

\section{AFTERWORD}

The workshop, with its opportunities to meet with a distinguished set of international scholars, to celebrate Krzysztof's Professorship, and to enjoy the elegant hospitality of the Institute for Mathematics at Bedlewo, were real highpoints of 2004 for WGH.

[1] R. S. Lakes, "Foam Structures with a Negative Poisson's Ratio", Science 235, 1038 (1987).

[2] A. Alderson and K. E. Evans, "Molecular Origin of Auxetic Behavior in Tetrahedral Framework Silicates", Phys. Rev. Lett. 89, 225503 (2002).

[3] K. W. Wojciechowski, "Remarks on Poisson's Ratio Beyond the Limits of Elasticity Theory", J. Phys. Soc. Japan 72, 1819 (2003).

[4] W. G. Hoover, "Molecular Dynamics and Irreversibility, from Boltzmann to Nosé, Trends in Chemical Physics 1, 33-54 (1991).

[5] R. E. Duff, W. H. Gust, E. B. Royce, M. Ross, A. C. Mitchell, R. N. Keeler, and W. G. Hoover, "Shockwave Studies in Condensed Media", pp. 397-406 in Behaviour 
of Dense Media under High Dynamic Pressures (Proceedings of the Paris Symposium of September 1967) Gordon and Breach, NY, 1968.

[6] D. R. Squire, A. C. Holt, and W. G. Hoover, "Isothermal Elastic Constants for Argon. Theory and Monte Carlo Calculations", Physica 42 388-397 (1969).

[7] W. G. Hoover, A. C. Holt, and D. R. Squire, "Adiabatic Elastic Constants for Argon. Theory and Monte Carlo Calculations", Physica 44, 437-443 (1969).

[8] W. G. Hoover, A. J. De Groot, C. G. Hoover, I. F Stowers, T. Kawai, B L Holian, T. Boku, S. Ihara, and J. Belak, "Large-Scale Elastic-Plastic Indentations Simulations via Nonequilibrium Molecular Dynamics", Phys. Rev. A 42, 5844-5853 (1990).

[9] W. G. Hoover, A. J. De Groot, and C. G. Hoover, Massively-Parallel Computer Simulation of Plane-Strain Elastic-Plastic Flow via Nonequilibrium Molecular Dynamics and Lagrangian Continuum Mechanics", Computers in Physics 6(2) 155-167 (1992). The threedimensional indentation simulations of Fig. 2 are taken from the cover of this issue.

[10] W. G. Hoover, C. G. Hoover, I. .F Stowers, A. J. De Groot, and B. Moran, "Simulation of Mechanical Deformation via Nonequilibrium Molecular Dynamics", pp. 315-322 in Microscopic Simulations of Complex Flows (Symposium held in Brussels, August 1989, a NATO Advanced Science Institute Series. Plenum, New York, 1990)

[11] S. M. Foiles, M. I. Baskes, and M. S. Daw, "EmbeddedAtom-Method Functions for the Face-Centered-Cubic Metals, $\mathrm{Cu}, \mathrm{Ag}, \mathrm{Au}, \mathrm{Ni}, \mathrm{Pd}, \mathrm{Pt}$, and their Alloys", Phys. Rev. B 33, 7983 (1986).

[12] W. G. Hoover, See Sec. II.B, Molecular Dynamics (Lecture Notes in Physics 258. Springer-Verlag, Berlin, 1986).

[13] W. G. Hoover, See page 189, Computational Statistical Mechanics (Elsevier, Amsterdam, 1991). This book is now out of print, but has been made available in electronic form at http://www.williamhoover.info.

[14] J. L. Lin, "DYNA3D: A Nonlinear, Explicit, ThreeDimensional Finite Element Code for Solid and Structural Mechanics", LLNL, Livermore, California (February 2004). The "User's Manual" is an electronic file which can be obtained from the United States Government's Energy Sciences Software Center at Oak Ridge, Tennessee.

FIG. 1. Auxetic copper foam, as shown in Ref. 1.

FIG. 2. Indentation pits, for the adiabatic motion of both smooth and atomistic indentors, as detailed in Ref. 9.

FIG. 3. Cutting of pair potential (at the left) and metallic embedded-atom potential (at the right) crystals using nonequilibrium molecular dynamics, as described in Ref. 10. Particles with above-average energies are shaded.
FIG. 4. Prototypical cellular structures, built up from shell elements (on the left) and brick elements (on the right). The external skin of elements, to which boundary forces are applied, is not shown here.

FIG. 5. The piecewise-linear dependence of acceleration on time used to deform and stress shell-element and brick-element structures. The amplitude of the final deformation is 100 times smaller than that of the initial inelastic deformation. The integrated velocity and displacement correspond to this acceleration function are also shown.

FIG. 6. Deformed auxetic structure, composed of 208,896 shell elements. The undeformed structure is shown at the left side of Fig. 4. Here the outermost "skin" of surrounding shell elements, to which the loads were applied, is not shown.

FIG. 7. Deformed structure (not auxetic), made up of 32,851 brick elements. The undeformed structure is shown at the right side of Fig. 4. Here the outermost skin of surrounding brick elements, to which the loads were applied, is not shown.

FIG. 8. The partition of a $65 \times 65 \times 65$ node problem over 32 processors. Nodes belonging to elements on the boundaries of the processors' partitions are shared between the corresponding processors. Here the external skin of elements is shown.

FIG. 9. Longitudinal and transverse displacements of a (typical) boundary node for the structure shown in Fig. 10 At the left, with an effective Poisson's Ratio of -0.1 , the initial random displacments were five times larger than those generating the more-negative effective Poisson's Ratio of -0.4 shown at the right. See the text for details.

FIG. 10. The structure corresponding to the displacements shown in Fig. 9. Young's modulus, the yield strength, and Poisson's ratio were equal to $100,0.10$, and 0.25 , with compressive strains of 0.10 in the $x, y$, and $z$ directions. The external skin of shell elements is not shown here. The total number of shell elements in the structure is 52,224 .

FIG. 11. Deformation of a structure made up of 259,712 brick elements. Here the external loads were applied directly to the outer surfaces shown in the figure, with no external skin. Young's modulus, the yield strength, and Poisson's ratio were equal to $100,0.10$, and 0.25 , with compressive strains of 0.20 in the $x, y$, and $z$ directions. The behavior of this structure is not auxetic. 


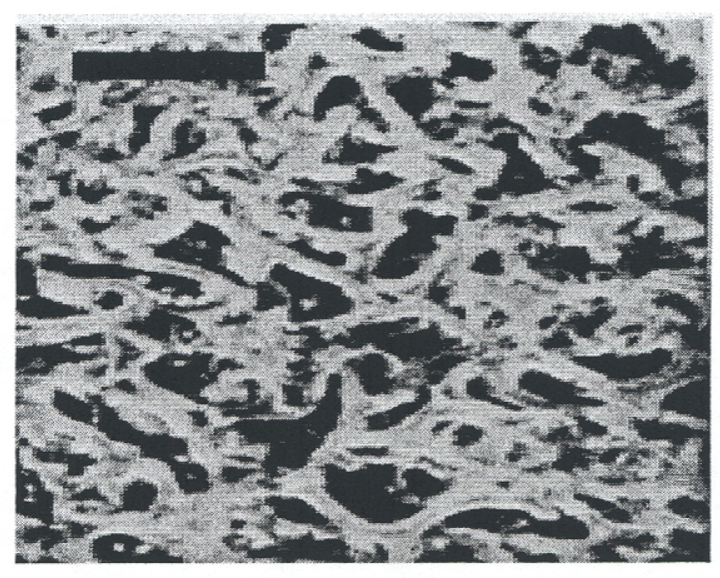

Figure 1 


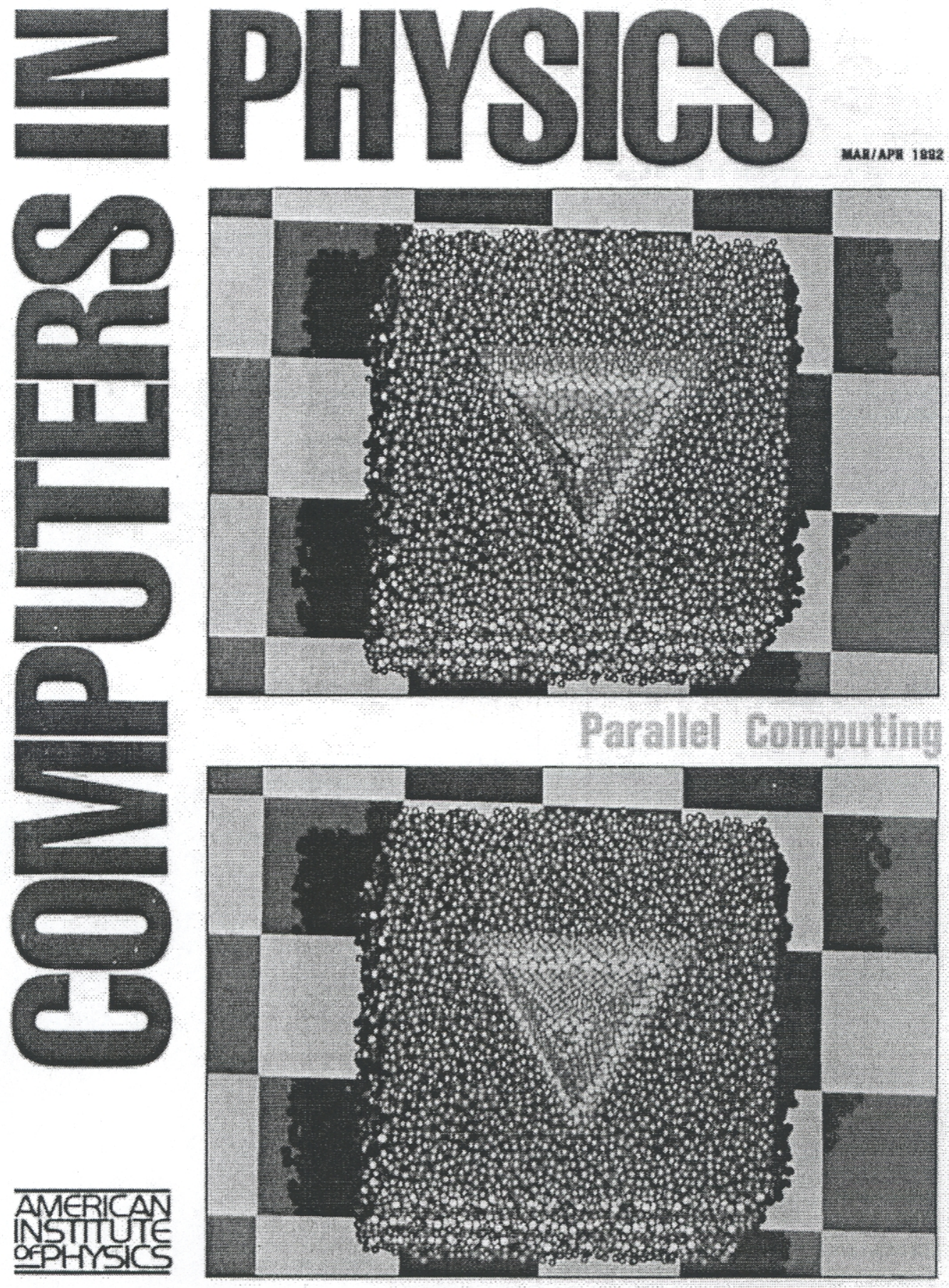

Figure 2 


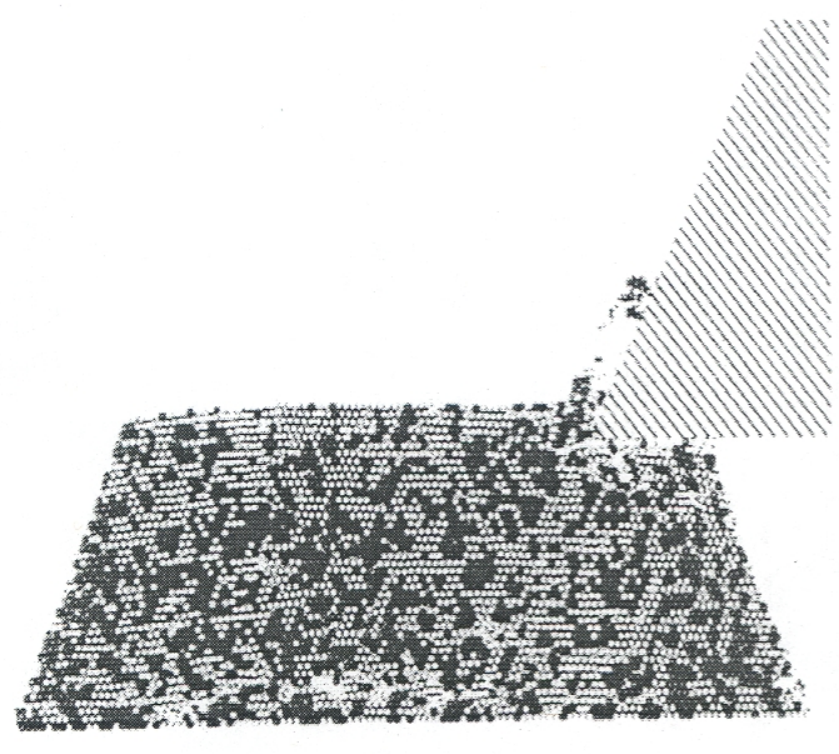

Lennard-Jones Crystal

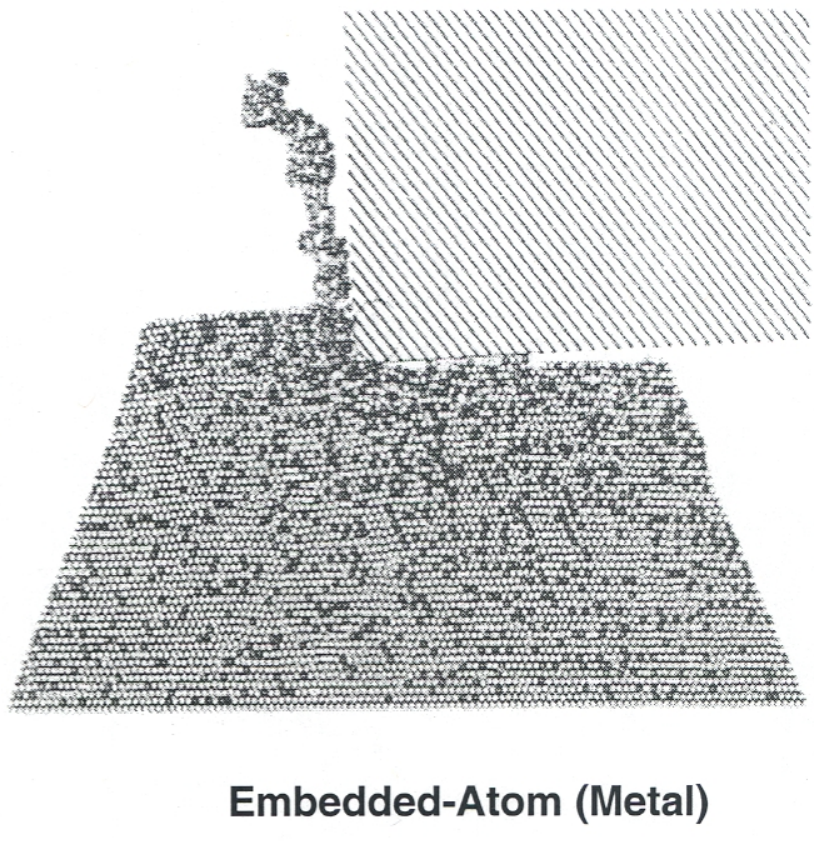

Figure 3 


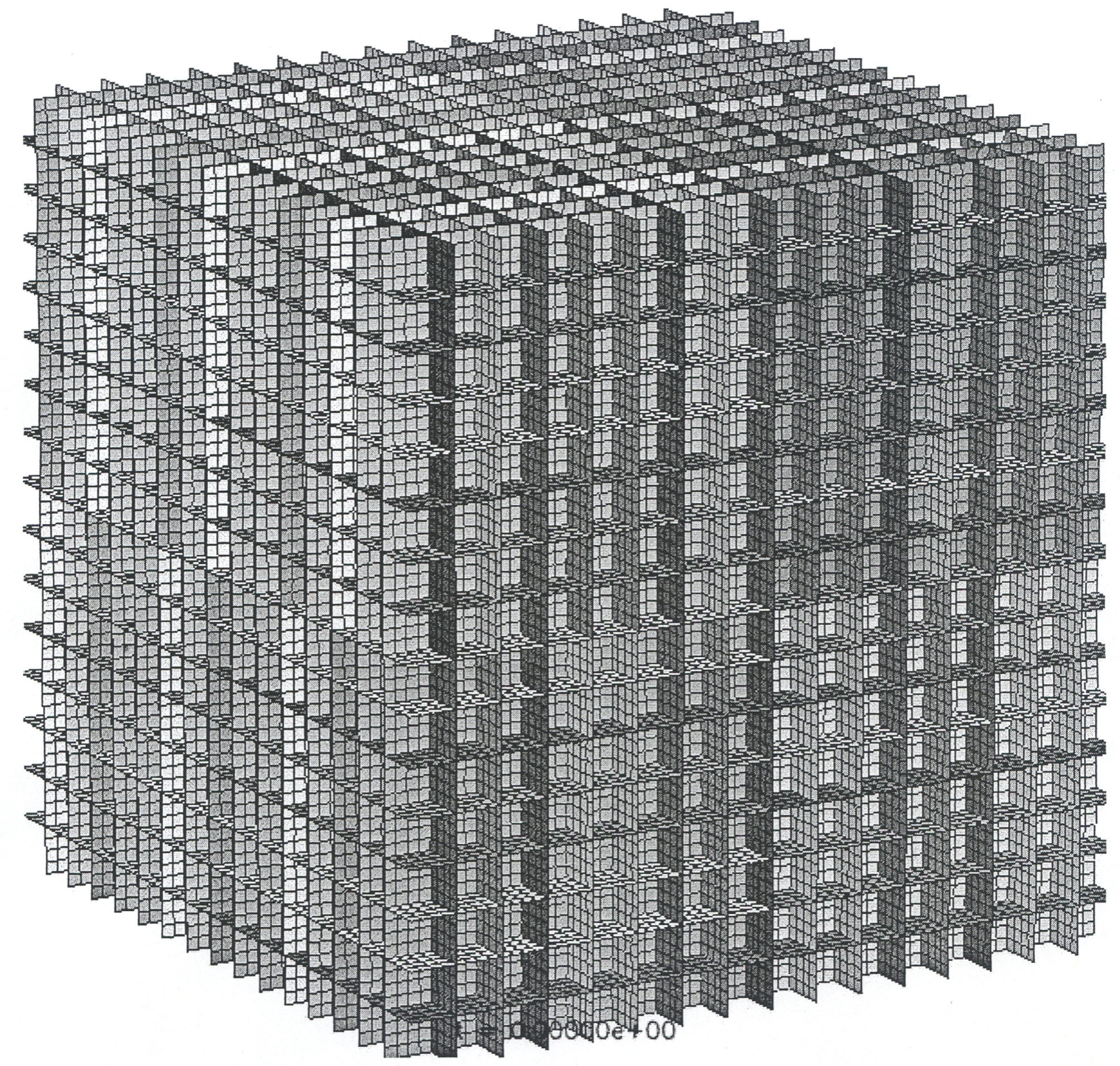

Figure 4 (left) 


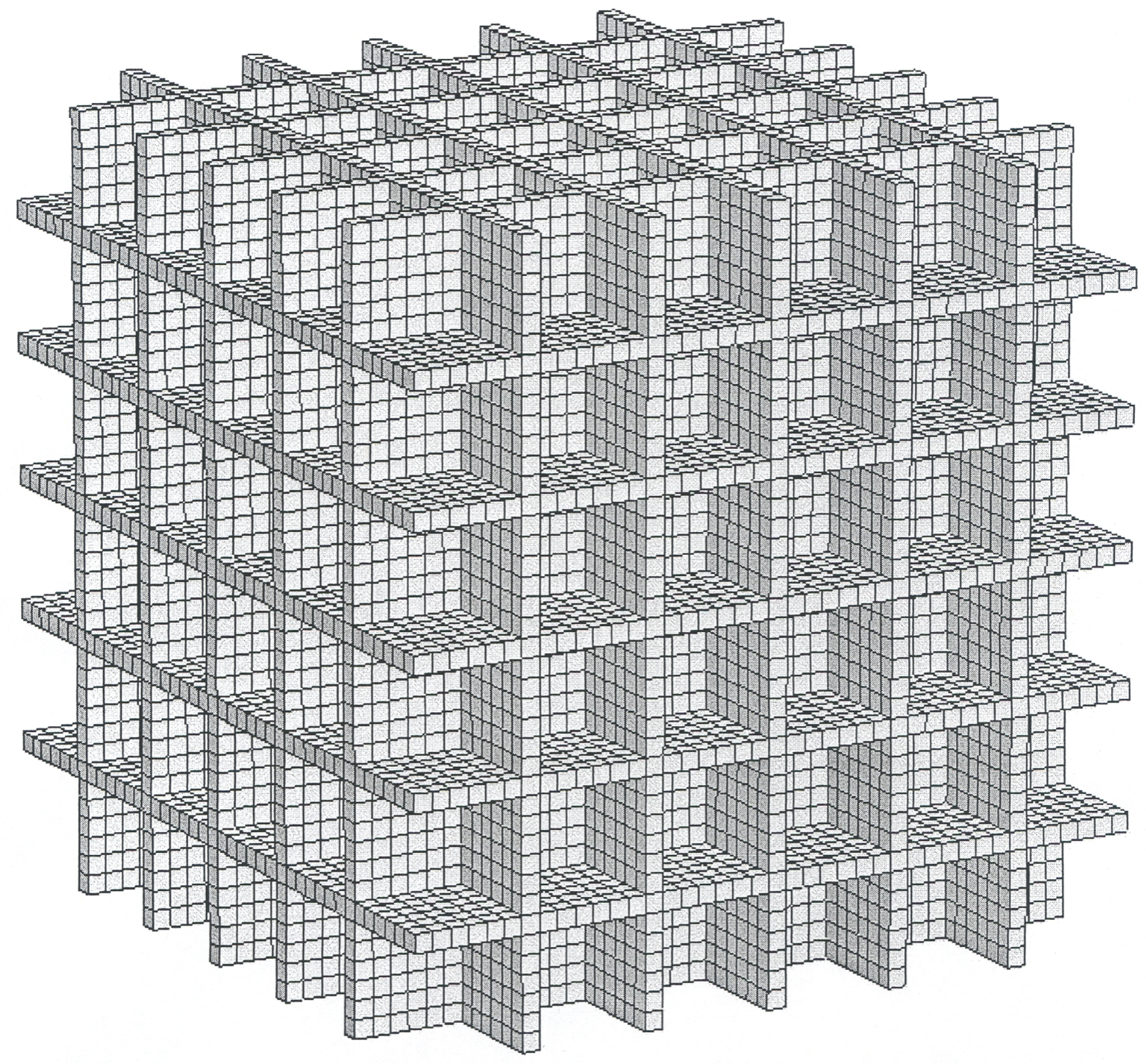

Figure 4 (right) 


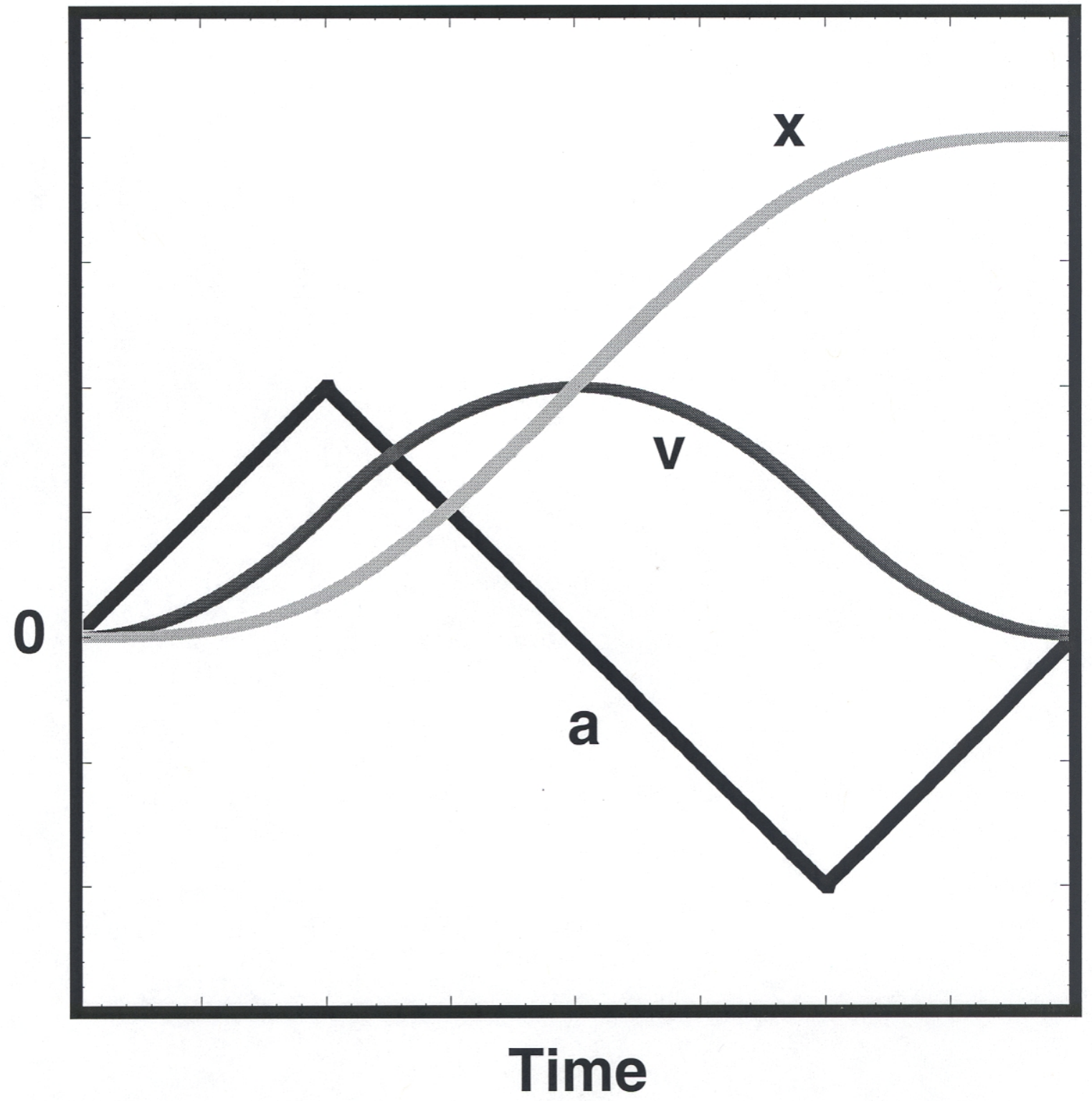

Figure 5 


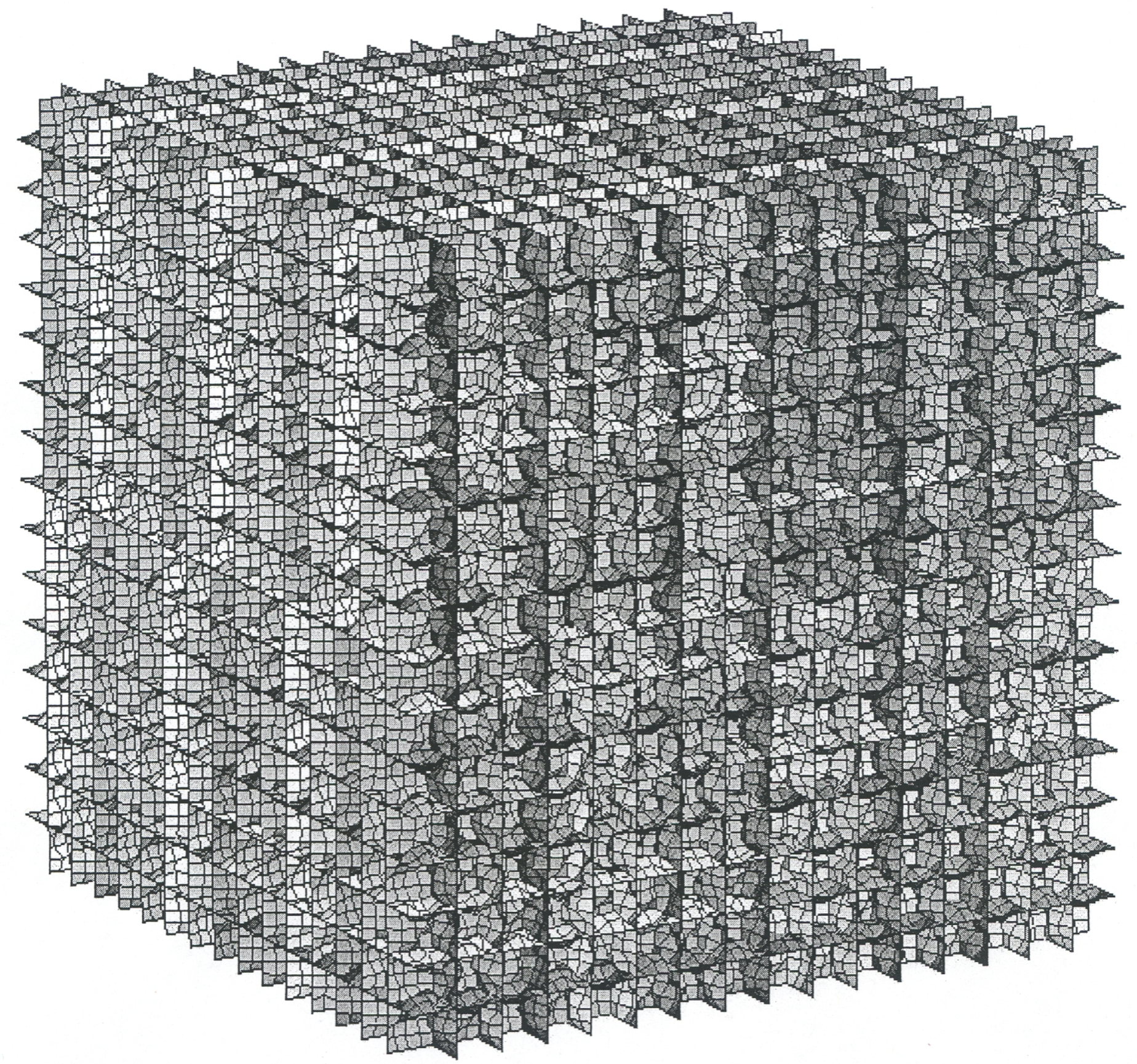

Figure 6 


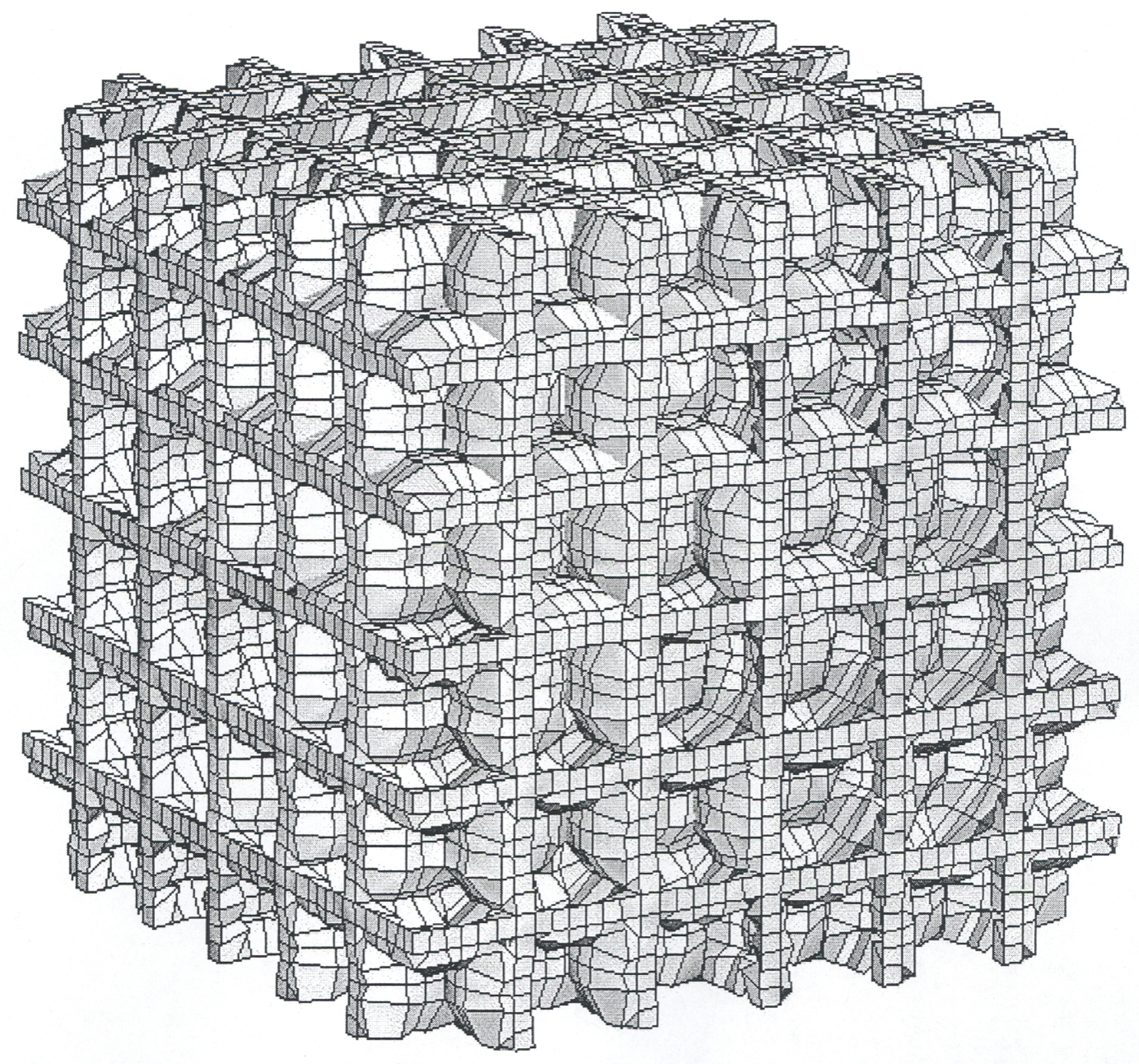

Figure 7 


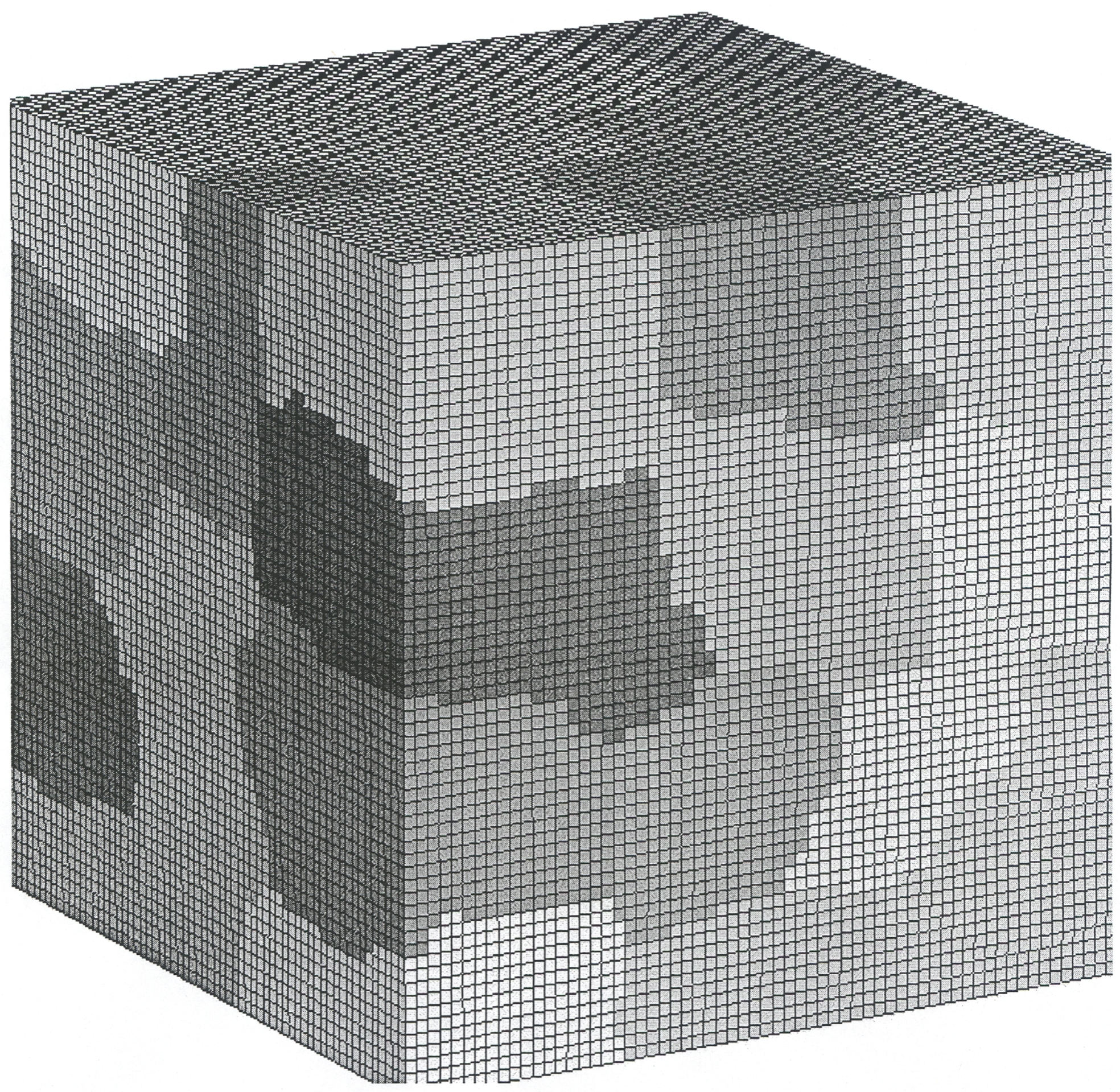

Figure 8 

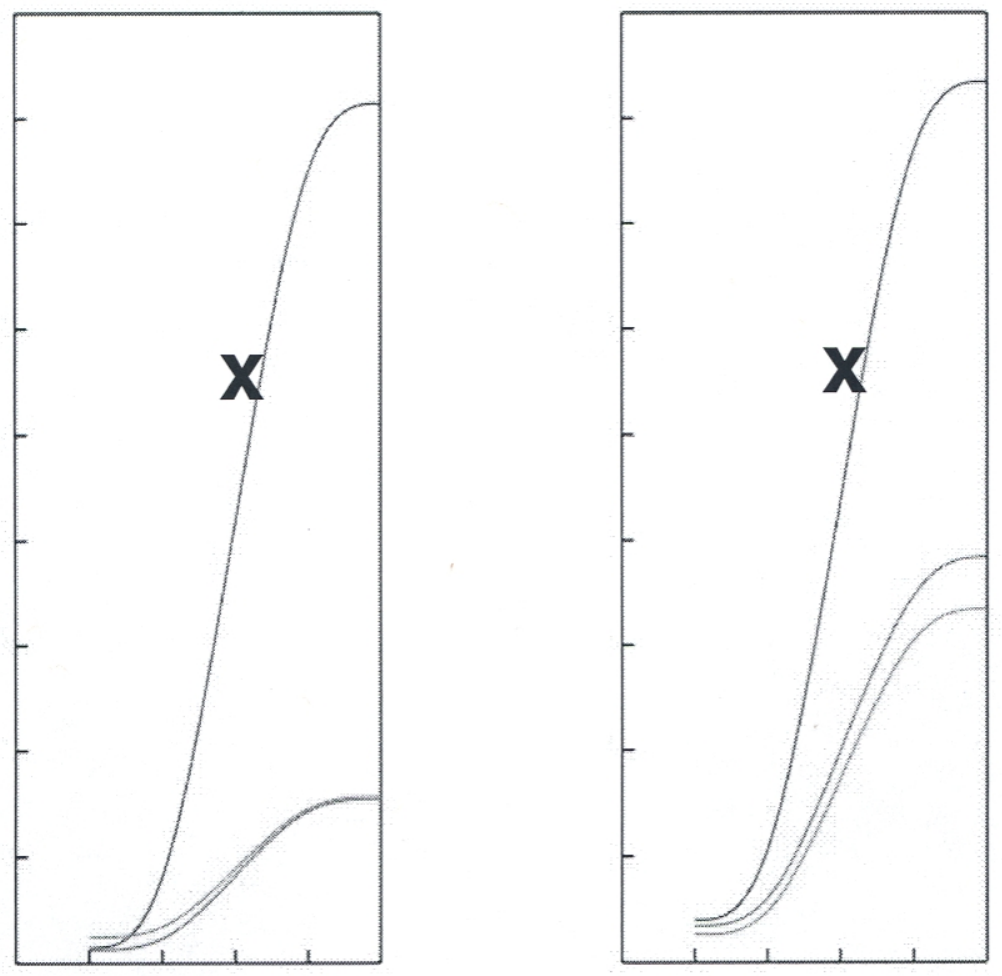

Time

Figure 9 


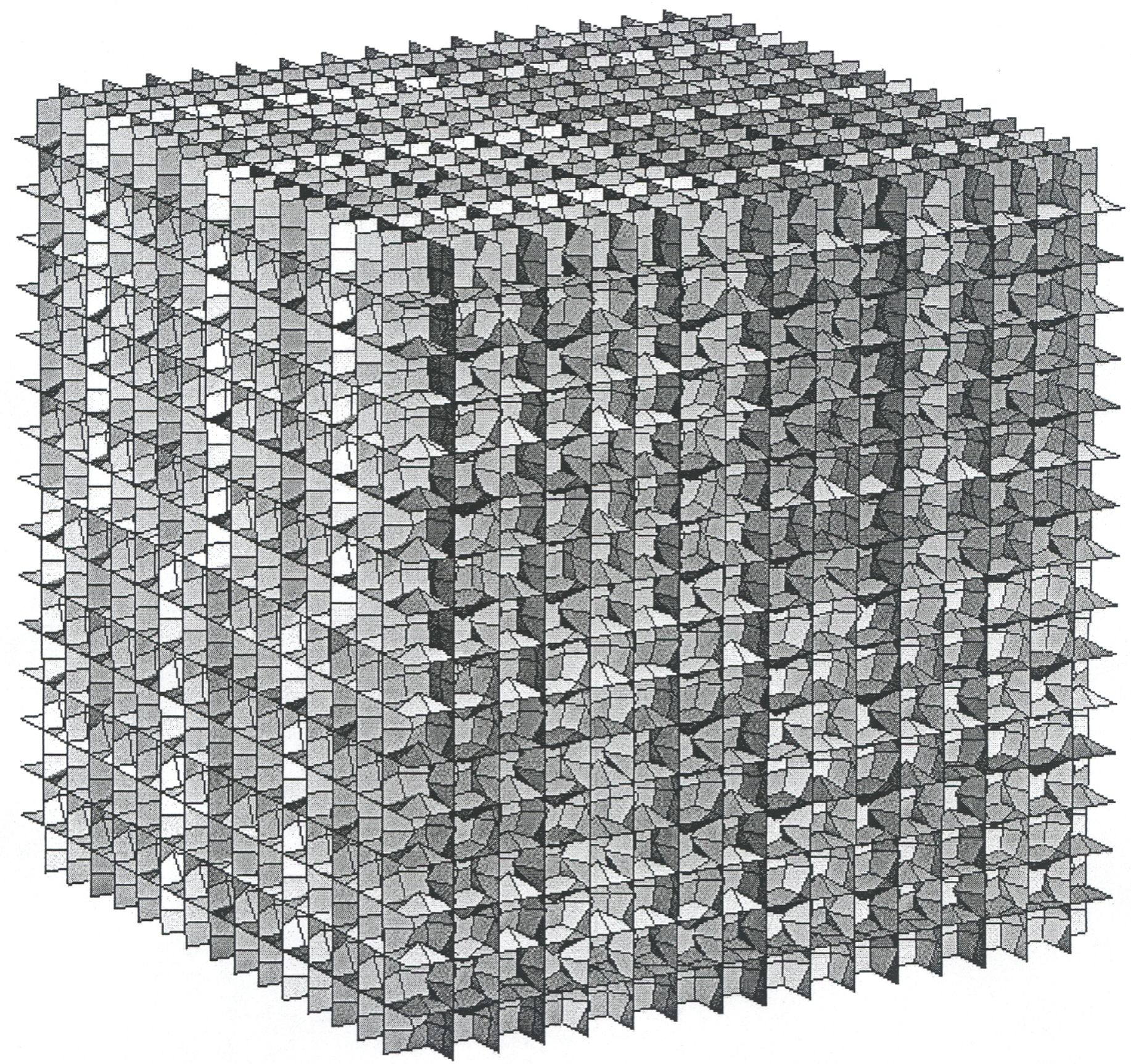

Figure 10 


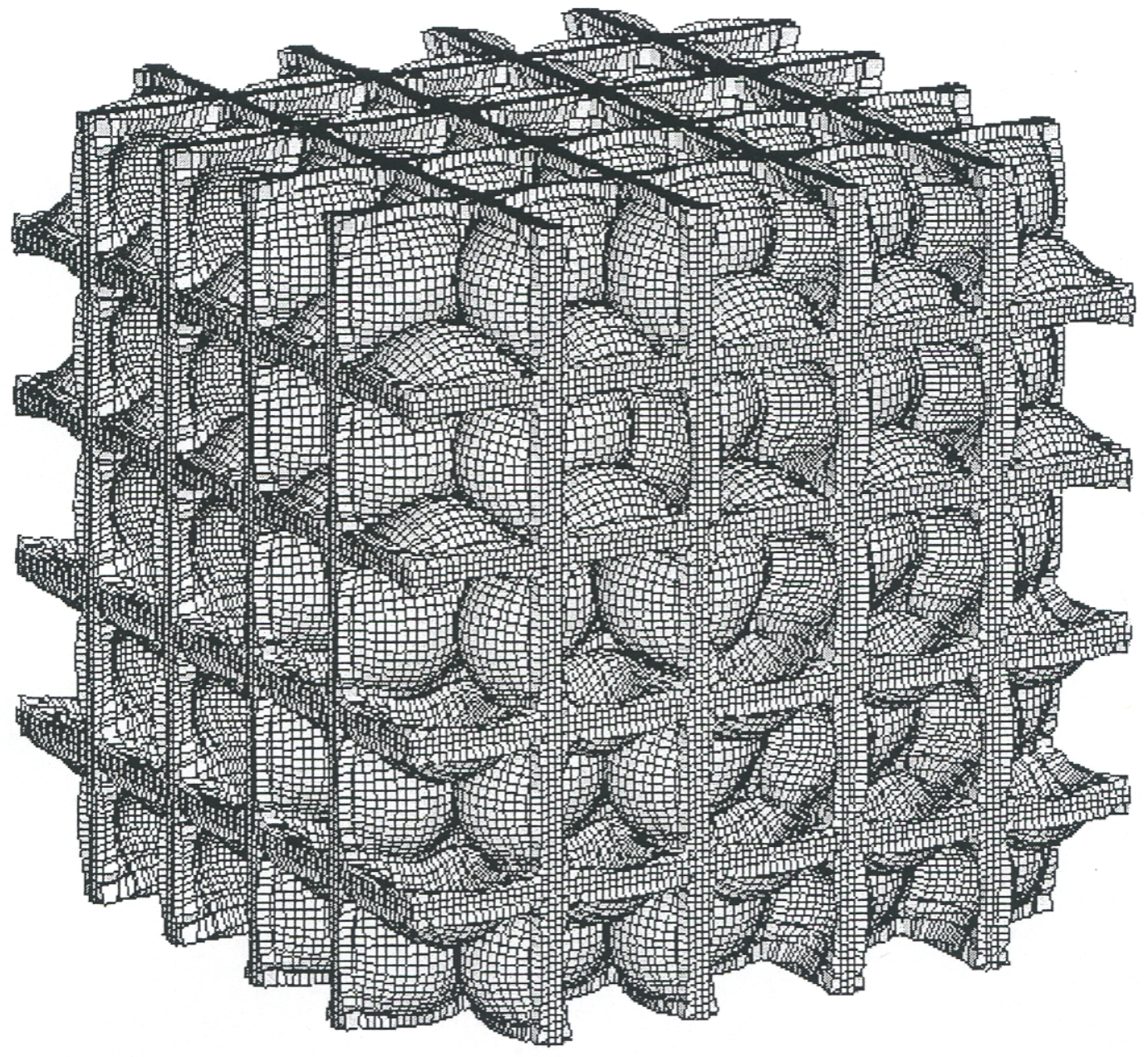

Figure 11 